\title{
Relation of Serum Plasmalogens and APOE Genotype to Cognition and Dementia in Older Persons in a Cross-Sectional Study
}

\author{
Dayan B. Goodenowe * and Vijitha Senanayake \\ Prodrome Sciences Inc., 204-2366 Avenue C N, Saskatoon, SK S7L 5X5, Canada; \\ v.senanayake@prodromesciences.com \\ * Correspondence: d.goodenowe@prodromesciences.com; Tel.: +1-306-500-4968
}

Received: 1 March 2019; Accepted: 21 April 2019; Published: 24 April 2019

\begin{abstract}
Using a community sample of 1205 elderly persons, we investigated the associations and potential interactions between Apolipoprotein $\mathrm{E}(A P O E)$ genotype and serum phosphatidylethanolamine (PlsEtn) on cognition and dementia. For each person, APOE genotype, PlsEtn Biosynthesis value (PBV, the combination of three key PlsEtn species), cognition (the combination of five specific cognitive domains), and diagnosis of dementia was determined. APOE genotype and PBV were observed to be non-interacting $(p>0.05)$ and independently associated with cognition: $A P O E$ (relative to $\varepsilon 3 \varepsilon 3: \varepsilon 2 \varepsilon 3$ $\left(\right.$ Coef $\left.=0.14, p=4.2 \times 10^{-2}\right) ; \varepsilon 3 \varepsilon 4 / \varepsilon 4 \varepsilon 4\left(\right.$ Coef $\left.=-0.22, p=6.2 \times 10^{-5}\right) ;$ PBV $\left(\right.$ Coef $\left.=0.12, p=1.7 \times 10^{-7}\right)$ and dementia: $A P O E$ (relative to $\varepsilon 3 \varepsilon 3: \varepsilon 2 \varepsilon 3$ (Odds Ratio OR $=0.44, p=3.0 \times 10^{-2}$ ); $\varepsilon 3 \varepsilon 4 / \varepsilon 4 \varepsilon 4(\mathrm{OR}=2.1$, $\left.\left.p=2.2 \times 10^{-4}\right)\right)$; PBV $\left(\mathrm{OR}=0.61, p=3.3 \times 10^{-6}\right)$. Associations are expressed per standard deviation (SD) and adjusted for serum lipids and demographics. Due to the independent and non-interacting nature of the APOE and PBV associations, the prevalence of dementia in $A P O E \varepsilon 3 \varepsilon 4 / \varepsilon 4 \varepsilon 4$ persons with high PBV values ( $>1 \mathrm{SD}$ from mean) was observed to be the same as $A P O E \varepsilon 3 \varepsilon 3$ persons ( $14.3 \%$ versus $14.0 \%$ ). Similarly, the prevalence of dementia in $A P O E \varepsilon 3 \varepsilon 3$ persons with high PBV values was only $5.7 \%$ versus $6.7 \%$ for $A P O E \varepsilon 2 \varepsilon 3$ persons. The results of these analyses indicate that the net effect of $A P O E$ genotype on cognition and the prevalence of dementia is dependent upon the plasmalogen status of the person.
\end{abstract}

Keywords: Alzheimer's disease; dementia; plasmalogen; APOE; cognition; cholesterol

\section{Introduction}

Apolipoprotein $\mathrm{E}(A P O E)$ is the predominant lipoprotein in the brain [1] and is the strongest genetic risk factor for Alzheimer's disease (AD) [2]. APOE has three common genetic variants $(\varepsilon 2$, $\varepsilon 3, \varepsilon 4)$ from which four allelic combinations make up approximately $95 \%$ of the U.S. population [3]. The AD risk gradient observed across the APOE genotypes is remarkable in that the percentage of subjects over the age of 60 with AD ranges from $\sim 3 \%$ in $\varepsilon 2 \varepsilon 3$ to $5 \%$ in $\varepsilon 3 \varepsilon 3$ to $18 \%$ in $\varepsilon 3 \varepsilon 4$ to $70 \%$ in $\varepsilon 4 \varepsilon 4$ subjects. The potential biological mechanisms responsible for the association of APOE genotype with AD risk are poorly understood [4,5].

Recent advances in AD research suggest that lipid abnormalities in the brain start in preclinical stages and are linked with neurodegeneration [6,7]. Lipids play critical structural and physiological roles in the brain [8]. Among phospholipids, glycerophospholipids containing a vinyl ether bond called plasmalogens play a disproportionately larger role in maintaining optimal brain function $[9,10]$. The deficiency of plasmalogen in brain and blood in AD has been reported by numerous researchers including us, and the association of plasmalogen deficiency with AD is well-accepted in the scientific community [6,7,11-14]. Furthermore, in AD, brain plasmalogen ethanolamine (PlsEtn) levels are reportedly lower than age-matched controls $[11,13,14]$, and low brain levels correlate with low serum 
levels [13]. Serum PlsEtn levels have been found to correlate with cognition in subjects with AD, including subjects pathologically confirmed by autopsy, and in serum samples collected at time of death in subjects with post-mortem AD pathology [15]. The decline in peroxisomal function is considered, at least, partially responsible for this plasmalogen depletion [15]. Moreover, lower peroxisomal $\beta$-oxidation, a key metabolic system involved in PlsEtn biosynthesis, is associated with lower cognition [16,17]. The role of PlsEtn in health and disease has been recently reviewed in detail [12].

Cholesterol [18,19] and brain cholesterol efflux [20] also play a significant role in the pathogenesis of AD. High Density Lipoprotein (HDL)-mediated cholesterol efflux (HDL-MCE) is a key component of cholesterol homeostasis and involves essentially two steps: intracellular cholesterol regulation and export and extracellular HDL binding and transport. Both PlsEtn and APOE have been shown to affect this system: PlsEtn deficient cells exhibit disrupted intracellular cholesterol transport and reduced cholesterol esterification which is restored by either genetic or metabolic restoration of PlsEtn [21]; increasing membrane Polyunsaturated fatty acid (PUFA) bearing PlsEtn concentrations increase cholesterol esterification via increased sterol-O-acetyltransferase (SOAT) and decreased total cholesterol [22]; low PlsEtn levels directly affect HDL-MCE, which is restored when PlsEtn levels are increased [23]; the potency of APOE-HDL particles to bind and remove cholesterol from neurons is isoform-dependent $(\varepsilon 2>\varepsilon 3>\varepsilon 4)$ [20]; synaptic cholesterol levels are isoform-dependent $(\varepsilon 4>\varepsilon 3>$ $\varepsilon 2)[24,25]$; and differential binding affinities of the $A P O E$ isoforms to the low-density lipoprotein receptor (LDLR) simultaneously affect cortical cholesterol levels and cognitive function [26].

Therefore, both PlsEtn [23] and ApoE [20] seem to play a role in cholesterol homeostasis and efflux, which could be potential mechanisms by which these variables affect AD pathogenesis. Since PlsEtn and ApoE appear to affect membrane cholesterol via independent mechanisms, we hypothesized that if membrane cholesterol dynamics are involved upstream of cognition and AD, then the PlsEtn and ApoE associations with cognition and odds of AD would interact. However, if membrane cholesterol changes and cognition are downstream effects of AD pathology, then no interaction should be observed. To test this hypothesis, we investigated the associations between APOE genotype, serum PlsEtn levels, and serum lipids, such as HDL, on cognition and odds of AD in a well-characterized community-based group of 1205 elderly persons enrolled in the Religious Orders Study (ROS) [27] or Rush Memory and Aging Project (MAP) [28].

\section{Materials and Methods}

\subsection{Participants}

Participants were selected from living subjects currently enrolled in the Religious Orders Study (ROS) or Rush Memory and Aging Project (MAP) [27,28] The Religious Orders Study began in 1994 and enrolls older Catholic clergy from across the USA and the Rush Memory and Aging Project began in 1997 and enrolls older lay persons from across northeastern Illinois. Both studies enroll persons without known dementia who agree to annual clinical evaluation. Both studies were approved by the Institutional Review Board of Rush University Medical Center and participants in both studies signed an informed consent. Information on ethics board approvals can be found in reports published by the ROS and MAP study teams $[27,29]$. The studies were conducted by the same investigative team and have a larger common core that allowed efficient merging of data for analyses [30]. We tested 1205 participants who had $A P O E$ genotyping data available.

\subsection{Clinical Evaluation}

A uniform structured clinical evaluation was performed to document the level of cognition, and the presence of dementia, $\mathrm{AD}$, mild cognitive impairment $(\mathrm{MCI})$, and other causes of cognitive impairment as previously described [16,31]. Dementia diagnosis was made separately, and a dementia diagnosis includes dementia due to other neurological diseases, in addition to AD. Therefore, not all subjects in the dementia category were included in the AD category. Dementia diagnosis was based 
on current diagnostic evaluation for dementia according to the Diagnostic and Statistical Manual of Mental Disorders (DSM-III-R) definition of dementia [17]. AD diagnosis was made according to the National Institute of Neurological and Communicative Disorders and Stroke/Alzheimer's Disease and Related Disorders Association (NINDCS/ADRDA) criteria [16]. The diagnosis of AD required a history of cognitive decline and evidence of impairment in memory and other cognitive abilities. Each study had a battery of 21 tests (Mini-Mental State Examination, Logical Memory Ia, Logical Memory IIa, Immediate story recall, Delayed story recall, Word List Memory, Word List Recall, Word List Recognition, Complex Ideational Material, Boston Naming Test, Category Fluency, National Adult Reading test, Digit Span Forward, Digit Span Backward, Digit Ordering, Symbol Digit Modalities Test, Number Comparison, Stroop word reading, Stroop color naming, Judgment of Line Orientation, and Standard Progressive Matrices); 19 tests (except Mini-Mental State Examination and Complex Ideational Material) were used to create a composite measure of global cognition (GCOG) based on the average z-score using the means and standard deviations from the baseline visit [28]. The tests were also used to summarize cognition into five cognitive domains: episodic memory (Logical Memory Ia, Logical Memory IIa, Immediate story recall, Delayed story recall, Word List Memory, Word List Recall, and Word List Recognition), semantic memory (Boston Naming Test, Category Fluency and National Adult Reading test), working memory (Digit Span Forward, Digit Span Backward and Digit Ordering), perceptual speed (Symbol Digit Modalities Test, Number Comparison, Stroop word reading, and Stroop color naming), and visuospatial ability (Judgment of Line Orientation and Standard Progressive Matrices) [28]. Composite score and scores representing each cognitive domain were used for statistical analyses.

\subsection{Serum Biomarker Analyses}

Fresh serum from a serum separator tube was sent to Quest Diagnostics (Chicago, IL) for HDL-Cholesterol (HDL-C), Low Density Lipoprotein (LDL)-Cholesterol (LDL-C), triglyceride (TG), and total cholesterol (TC) levels.

Separate serum samples were stored at $-80{ }^{\circ} \mathrm{C}$ until thawed for analysis. Serum levels of phosphatidylethanolamine (PtdEtn) 16:0/22:6, PlsEtn 16:0/22:6, PlsEtn 18:0/20:5, and PlsEtn 16:0/22:4 were determined using stable isotope dilution liquid chromatography tandem mass spectrometry (LC-MS/MS) using ${ }^{13} \mathrm{C}$-PlsEtn 16:0/22:6 and ${ }^{13} \mathrm{C}-\mathrm{PtdEtn}$ 16:0/22:6. $10 \mu \mathrm{L}$ of internal standards $(1 \mu \mathrm{g} / \mathrm{mL})$, $40 \mu \mathrm{L}$ of $4 \%$ formic acid in water and $0.5 \mathrm{~mL}$ of $100 \%$ ethyl acetate (EtOAc) were added to $20 \mu \mathrm{L}$ of serum. The mixture was vortexed for $15 \mathrm{~min}$ and centrifuged at $4{ }^{\circ} \mathrm{C}$ for $2 \mathrm{~min}$ at $1400 \mathrm{rpm}$. The supernatant was used for the analysis of each sample. All extracts were stored at $-80{ }^{\circ} \mathrm{C}$ until analysis. $100 \mu \mathrm{L}$ of the EtOAc fraction was injected by flow injection at $600 \mu \mathrm{L} / \mathrm{min}$ and ionized using negative atmospheric pressure chemical ionization (APCI) mode using a triple quadrupole mass spectrometer (IONICS 3Q, Ionics Mass Spectrometry Group, Bolton, ON, Canada) coupled to a Gilson GX-271 (Gilson Inc., Middleton, WI, USA). Parent (M-H): fragment (sn2 fatty acid) transitions were monitored for each analyte in the multiple reaction monitoring (MRM) mode. Standard curves were generated using ${ }^{12}$ C-PlsEtn 16:0/22:6 and ${ }^{12}$ C-PtdEtn 16:0/22:6 and encompassed the full biological range observed, $r^{2}>0.98$. All standards and stable isotopes were $>95 \%$ pure and manufactured by Phenomenome Discoveries Inc., (Saskatoon, SK, Canada).

A Plasmalogen Biosynthesis value (PBV) was created for each participant by calculating and combining three PlsEtn/PlsEtn and two PlsEtn/PtdEtn ratios. As explained below, the PlsEtn16:0/22:6 to PtdEtn16:0/22:6 and PlsEtn18:0/20:5 to PtdEtn16:0/22:6 ratios were used to assess the relative PlsEtn and PtdEtn pool sizes; and the PlsEtn16:0/22:6 to PlsEtn16:0/22:4, PlsEtn18:0/20:5 to PlsEtn16:0/22:4, and PlsEtn 18:0/20:5 to PlsEtn16:0/22:6 ratios were used to assess peroxisomal $\beta$-oxidation. These molecules were selected based on their sn-2 fatty acids. These fatty acids are either metabolized by peroxisomal $\beta$-oxidation (22:4 and 22:6) [32] or are a product of peroxisomal $\beta$-oxidation $(20: 5$ or $22: 6)[33,34]$ and are also known to be associated with AD [35,36]. PlsEtn and PtdEtn species used in these ratios are among the most abundant PlsEtn and PtdEtn species in the blood [37], thus, PlsEtn to PtdEtn ratio 
represents their relative abundance [38]. For the calculation of ratios, each ratio was divided by its sex-specific mean, and then $\log 10$ transformed to equally weight each ratio, after which these five values were averaged to create the PBV.

\subsection{APOE Genotyping}

Participants were genotyped for APOE alleles by Polymorphic DNA Technologies. Participants were grouped according to APOE genotype as follows: $\varepsilon 2 \varepsilon 3(n=149), \varepsilon 3 \varepsilon 3(n=745)$, and $\varepsilon 3 \varepsilon 4$ or $\varepsilon 4 \varepsilon 4$ $(\varepsilon 3 \varepsilon 4 / \varepsilon 4 \varepsilon 4$, total $n=269)$. Participants with an APOE genotype of $\varepsilon 2 \varepsilon 2(n=4)$ or $\varepsilon 2 \varepsilon 4(n=16)$ were excluded from the analyses.

\subsection{Statistical Analyses}

In descriptive analyses, males and females were compared using chi-square or Fishers's exact tests for categorical variables and Wilcoxon rank-sum tests for continuous variables. To reduce skewness, TG, TC, HDL-C, LDL-C, HDL ratio (HDL-R) (HDL-C/TC) were log10 transformed. For continuous outcomes, such as GCOG, we first screened potential predictors by examining correlations with the outcome and then fit multiple linear regression models including both variables of interest (PBV, HDL-R, APOE $\varepsilon 2 \varepsilon 3, A P O E \varepsilon 3 \varepsilon 4 / \varepsilon 4 \varepsilon 4$ ) with terms to adjust for age, sex, and education. The predictors that passed the screening were then included in a single multiple regression model, with terms for age, sex, and education. For continuous predictors, associations are shown per standard deviation (SD) of the predictor. We augmented the model by including the interactions of $A P O E$ genotypes with PBV; we also repeated linear regression models in stratified $A P O E \varepsilon 2 \varepsilon 3, A P O E \varepsilon 3 \varepsilon 3$, and $A P O E \varepsilon 3 \varepsilon 4 / \varepsilon 4 \varepsilon 4$ groups. For categorical variables, we used a similar approach: we first ran a Pearson chi-square or a Fisher's Exact test to determine the presence of association with various covariates, followed by multiple logistic regression. For the determination of associations with AD in the presence of MCI, ordinal logistic regression was performed. Results are reported as Odds Ratio (OR) or Coefficient (coef), $p$ value. Stata 13.1 was used to perform all analyses; statistical significance is $p<0.05$ in all analyses.

\section{Results}

\subsection{Demographics}

Participant demographic characteristics and the number of people in each diagnostic category are given in Tables 1 and 2.

Table 1. Demographic and serum lipid summary by gender.

\begin{tabular}{cccccc}
\hline \multicolumn{7}{c}{ Female $(n=921)$} & Male $(n=284)$ \\
Variable & Mean & SD & Mean & SD & $p$-Value \\
Age (range) & $83.8(58.3-103.9)$ & 7.6 & $84.3(64.2-101.0)$ & 7.1 & $3.8 \times 10^{-1}$ \\
Education & 15.5 & 3.4 & 16.1 & 4.0 & $1.7 \times 10^{-2} *$ \\
& \multicolumn{7}{c}{ Serum Lipids } \\
Variable & Mean & SD & Mean & SD & $p$-Value \\
TG & 136.8 & 67.9 & 122.7 & 59.2 & 3.3 e $\times 10^{-3} *$ \\
TC & 192.6 & 38.3 & 161.7 & 35.6 & $6.0 \times 10^{-28 *}$ \\
HDL-C & 63.9 & 18.4 & 52.5 & 15.2 & $4.9 \times 10^{-18 *}$ \\
LDL-C & 101.4 & 33.2 & 84.6 & 29.1 & $1.9 \times 10^{-12} *$ \\
HDL-R & 0.338 & 0.092 & 0.331 & 0.088 & $3.5 \times 10^{-1}$ \\
& Serum Ethanolamine Glycerophospholipids & \\
Variable & Mean & SD & Mean & SD & $p$-Value \\
PE226 & 1.60 & 0.89 & 1.08 & 0.63 & $4.6 \times 10^{-19} *$ \\
PL224 & 0.85 & 0.48 & 0.72 & 0.31 & $8.0 \times 10^{-5} *$ \\
PL205 & 1.35 & 1.83 & 0.98 & 0.81 & $1.1 \times 10^{-3} *$ \\
PL226 & 3.48 & 2.28 & 2.84 & 1.22 & $8.5 \times 10^{-6} *$ \\
\hline
\end{tabular}


Table 1. Cont.

\begin{tabular}{|c|c|c|c|c|c|}
\hline & \multicolumn{2}{|c|}{ Female $(n=921)$} & \multicolumn{2}{|c|}{ Male $(n=284)$} & \\
\hline \multicolumn{6}{|c|}{ Plasmalogen Ratios } \\
\hline Variable & Mean & SD & Mean & SD & $p$-Value \\
\hline PL205/PE226 & 0.65 & 0.55 & 0.715 & 0.480 & $7.3 \times 10^{-2}$ \\
\hline PL226/PE226 & 1.81 & 0.88 & 2.18 & 1.00 & $1.6 \times 10^{-9 *}$ \\
\hline PL205/PL224 & 1.94 & 2.49 & 1.67 & 1.98 & $1.0 \times 10^{-1}$ \\
\hline PL226/PL224 & 4.55 & 2.36 & 4.39 & 2.27 & $2.9 \times 10^{-1}$ \\
\hline PL205/PL226 & 0.36 & 0.23 & 0.337 & 0.186 & $1.4 \times 10^{-1}$ \\
\hline PBV & -0.09 & 0.21 & -0.075 & 0.179 & $2.9 \times 10^{-1}$ \\
\hline
\end{tabular}

Mean and standard deviation of the demographic variables, lipoproteins and phospholipid are given per gender. Serum lipid values are given in $\mathrm{mg} / \mathrm{dL}$. Internal standard-normalized values are given for ethanolamine glycerophospholipids and plasmalogen ratios. TG: Triglycerides, TC: Total cholesterol; HDL-C; HDL cholesterol; LDL-C: LDL cholesterol, HDL-R: HDL cholesterol to Total cholesterol ratio; PE226: Phosphatidyl ethanolamine 16:0/22:6; PL224: Plasmalogen ethanolamine 16:0/22:4; PL205: Plasmalogen ethanolamine 18:0/20:5; PL226: Plasmalogen ethanolamine 16:0/22:6; PL205/PE226: ratio of Plasmalogen ethanolamine 18:0/20:5 to Phosphatidyl ethanolamine 16:0/22:6; PL226/PE226: ratio of Plasmalogen ethanolamine 16:0/22:6 to Phosphatidyl ethanolamine 16:0/22:6; PL205/PL224: ratio of Plasmalogen ethanolamine 18:0/20:5 to Plasmalogen ethanolamine 16:0/22:4; PL226/PL224: ratio of Plasmalogen ethanolamine 16:0/22:6 to Plasmalogen ethanolamine 16:0/22:4; PL205/PL226: ratio of Plasmalogen ethanolamine 18:0/20:5 to Plasmalogen ethanolamine 16:0/22:6; PBV: Averaged value of the five mean normalized (gender-based) $\log 10$ transformed plasmalogen ratios. For clarity, significant $p$ values $(p<0.05)$ are indicated by an asterisk*

Table 2. Clinical diagnoses and global cognition in key variable classes.

\begin{tabular}{cccccccc}
\hline & \multicolumn{9}{c}{ Clinical Diagnosis and Cognition by Key Variable Classes } & \\
Variable Class & All & No Dementia & Dementia (\%) & NCI & MCI & AD & GCOG Mean (SD) \\
\hline Female & 921 & 784 & $137(14.9)$ & 597 & 168 & 104 & $-0.025(0.829)$ \\
Male & 284 & 246 & $38(13.4)$ & 184 & 68 & 26 & $-0.051(0.756)$ \\
PBV > 1 SD & 183 & 172 & $11(6.0)$ & 143 & 26 & 9 & $0.238(0.647)$ \\
PBV \pm 1 SD & 851 & 740 & $111(13.0)$ & 556 & 177 & 80 & $-0.243(0.782)$ \\
PBV < 1 SD & 171 & 118 & $53(31.0)$ & 82 & 33 & 41 & $-0.374(0.996)$ \\
ApoE e2e3 & 149 & 139 & $10(6.7)$ & 116 & 24 & 3 & $0.178(0.589)$ \\
+ PBV > 1 SD & 25 & 25 & $0(0)$ & 23 & 2 & 0 & $0.370(0.442)$ \\
+ PBV \pm 1 SD & 106 & 100 & $6(5.7)$ & 82 & 18 & 2 & $0.184(0.612)$ \\
+ PBV < 1 SD & 18 & 14 & $4(22.2)$ & 11 & 4 & 1 & $-0.139(0.528)$ \\
ApoE e3e3 & 745 & 641 & $104(14.0)$ & 480 & 152 & 75 & $-0.028(0.817)$ \\
+ PBV > 1 SD & 122 & 115 & $7(5.7)$ & 96 & 17 & 5 & $0.262(0.606)$ \\
+ PBV \pm 1 SD & 511 & 448 & $63(12.3)$ & 330 & 113 & 42 & $-0.019(0.771)$ \\
+ PBV < 1 SD & 112 & 78 & $34(30.4)$ & 54 & 22 & 28 & $-0.406(1.063)$ \\
ApoE e3e4/e4e4 & 269 & 210 & $59(21.9)$ & 151 & 55 & 51 & $-0.181(0.900)$ \\
+ PBV > 1 SD & 28 & 24 & $4(14.3)$ & 18 & 6 & 4 & $-0.010(0.917)$ \\
+ PBV \pm 1 SD & 206 & 164 & $42(20.4)$ & 119 & 43 & 36 & $-0.173(0.881)$ \\
+ PBV < 1 SD & 35 & 22 & $13(37.1)$ & 14 & 6 & 11 & $-0.390(1.007)$ \\
\hline
\end{tabular}

Clinical diagnosis (NCI, MCI, and AD) and global cognition score (GCOG) according to gender, PBV levels and APOE allele composition. Note lesser number of AD cases and higher GCOG when PBV > 1 SD. SD: Standard deviation; NCI: No cognitive impairment; MCI: Mild cognitive impairment; AD: Alzheimer's disease; PBV: Plasmalogen Biosynthesis Value.

\subsection{Relation of PBV and APOE to AD and Level of Cognition}

The relation of PBV and APOE to the diagnosis of Alzheimer's disease (AD) and mild cognitive impairment $(\mathrm{MCI})$ versus no cognitive impairment $(\mathrm{NCI})$ was examined using an ordered logistic regression model. After adjusting for serum lipids and demographic measures, a higher serum PBV level was associated with a lower odds of MCI/AD (Table 3). Specifically, each 1 SD increase in PBV was associated with about a $30 \%$ reduced odds of MCI/AD. By contrast, an APOE $\varepsilon 2 \varepsilon 3$ was associated with about a $50 \%$ lower odds of MCI/AD, and an APOE $\varepsilon 3 \varepsilon 4 / \varepsilon 4 \varepsilon 4$ was associated with nearly twice the odds of MCI/AD (Table 3). 
Table 3. Multivariate association of clinical and biological variables with dementia, mild cognitive impairment/Alzheimer's disease (MCI/AD), and cognition.

\begin{tabular}{|c|c|c|c|c|c|c|c|c|}
\hline & Dementia & NCI-MCI-AD & Global Cognition & Episodic Memory & Visuospatial Ability & Perceptual Speed & Semantic Memory & Working Memory \\
\hline Variable & $\begin{array}{c}(n=1085) \\
\mathrm{OR}^{3}(p)\end{array}$ & $\begin{array}{c}(n=1030) \\
\mathrm{OR}^{2}(p)\end{array}$ & $\begin{array}{l}(n=1049) \\
\text { Coef. }^{1}(p)\end{array}$ & $\begin{array}{l}(n=1026) \\
\text { Coef. }^{1}(p)\end{array}$ & $\begin{array}{c}(n=992) \\
\text { Coef. }^{1}(p)\end{array}$ & $\begin{array}{l}(n=1005) \\
\text { Coef. }^{1}(p)\end{array}$ & $\begin{array}{l}(n=1022) \\
\text { Coef. }{ }^{1}(p)\end{array}$ & $\begin{array}{l}(n=1049) \\
\text { Coef. }^{1}(p)\end{array}$ \\
\hline & $0.607\left(3.3 \times 10^{-6}\right)^{*}$ & $0.699\left(2.5 \times 10^{-6}\right)^{*}$ & $0.118\left(1.7 \times 10^{-7}\right)^{*}$ & $0.134\left(5.3 \times 10^{-6}\right) *$ & $0.051\left(4.4 \times 10^{-2}\right)^{*}$ & $0.111\left(5.2 \times 10^{-5}\right)^{*}$ & $0.107\left(3.1 \times 10^{-5}\right)^{*}$ & $0.079\left(1.6 \times 10^{-3}\right)^{*}$ \\
\hline HDL-R $^{4}$ & $0.773(5.9 \times 1$ & $0.904\left(1.6 \times 10^{-1}\right)$ & $\left.0^{-3}\right)^{*}$ & $\left.0^{-3}\right)^{*}$ & $0.018\left(4.8 \times 10^{-1}\right)$ & $0.019\left(4.8 \times 10^{-1}\right)$ & $0.042\left(1.0 \times 10^{-1}\right)$ & $0.060(1.6$ \\
\hline & & & & & & & & \\
\hline$A P O E \varepsilon 2 \varepsilon 3$ & $0.442\left(3.0 \times 10^{-2}\right) *$ & $0.462\left(1.8 \times 10^{-3}\right)^{*}$ & $0.136\left(4.2 \times 10^{-2}\right)^{*}$ & $0.230\left(8.1 \times 10^{-3}\right)^{*}$ & $0.062\left(4.1 \times 10^{-1}\right)$ & $-0.033\left(5.2 \times 10^{-5}\right)^{*}$ & $0.112\left(1.4 \times 10^{-1}\right)$ & $0.048\left(5.2 \times 10^{-1}\right)$ \\
\hline$A P O E \varepsilon 3 \varepsilon 4, \varepsilon 4 \varepsilon 4$ & $2.125\left(2.2 \times 10^{-4}\right) *$ & $1.996\left(1.9 \times 10^{-5}\right) *$ & $217\left(6.2 \times 10^{-5}\right) *$ & $319\left(7.6 \times 10^{-6}\right)^{*}$ & $-0.076\left(2.2 \times 10^{-1}\right)$ & $-0.276\left(3.1 \times 10^{-5}\right) *$ & $-0.190\left(2.0 \times 10^{-3}\right)^{*}$ & $-0.105\left(7.9 \times 10^{-2}\right)$ \\
\hline Age * & $2.271\left(1.4 \times 10^{-13}\right)^{*}$ & $2.056\left(2.0 \times 10^{-19}\right)^{*}$ & $-0.262\left(4.1 \times 10^{-3}\right)^{*}$ & $-0.290\left(2.6 \times 10^{-22}\right)^{*}$ & $-0.137\left(8.6 \times 10^{-8}\right)^{*}$ & $-0.352\left(1.6 \times 10^{-35}\right) *$ & $-0.191\left(1.0 \times 10^{-13}\right)^{*}$ & $-0.177\left(1.9 \times 10^{-12}\right)^{*}$ \\
\hline Education * & $0.957\left(6.5 \times 10^{-1}\right)$ & $0.917\left(2.3 \times 10^{-1}\right)$ & $0.183\left(4.8 \times 10^{-16}\right)^{*}$ & $0.165\left(1.9 \times 10^{-8}\right)^{*}$ & $0.255\left(1.0 \times 10^{-22}\right)^{*}$ & $0.204\left(1.2 \times 10^{-13}\right)^{*}$ & $0.229\left(6.7 \times 10^{-19}\right)^{*}$ & $0.174\left(3.2 \times 10^{-12}\right)^{*}$ \\
\hline Female & & & & & & & & \\
\hline Male & $1.035\left(8.8 \times 10^{-1}\right)$ & $1.104\left(5.5 \times 10^{-1}\right)$ & $-0.048\left(3.7 \times 10^{-1}\right)$ & $-0.159\left(2.3 \times 10^{-2}\right)^{*}$ & $0.205\left(6.9 \times 10^{-4}\right)^{*}$ & $-0.156\left(1.6 \times 10^{-2}\right)^{*}$ & $-0.013\left(8.2 \times 10^{-1}\right)$ & $0.066\left(2.7 \times 10^{-1}\right)$ \\
\hline
\end{tabular}

$1.035\left(8.8 \times 10^{-1}\right)-1.104\left(5.5 \times 10^{-1}\right)-0.08\left(3.7 \times 10^{-1}\right)-0.159\left(23 \times 10^{-2}\right) * 0.205\left(6.9 \times 10^{-4}\right) *-0.156(1.6 \times 1$

odds of dementia (logistic regression), MCI/AD diagnosis (logistic regression) and cognitive scores (linear regression). ${ }^{1}$ Coefficients for continuous variables expressed per standard deviation (SD); multiple regression; ${ }^{2}$ Odds ratios for continuous variables expressed per SD, ordinal logistic regression with $\mathrm{AD} / \mathrm{MCI} / \mathrm{NCI}$ as the outcome; ${ }^{3}$ Odds ratios for continuous variables expressed per SD, multiple logistic regression; ${ }^{4} \mathrm{HDL}-\mathrm{R}$ was mean normalized and $\log _{10}$ transformed; ${ }^{5}$ Age and Education were mean centered. For clarity, significant $p$ values $(p<0.05)$ are indicated by an asterisk* 
Because clinical diagnoses represent cutoffs across a continuum of cognition, we next examined the relation of PBV and APOE to GCOG using a linear regression model. After adjusting for serum lipids and demographic measures, each 1 SD higher serum PBV level was associated with more than a $10 \%$ higher GCOG (Table 3). APOE $\varepsilon 2 \varepsilon 3$ also was associated with more than a $10 \%$ higher GCOG, whereas APOE $\varepsilon 3 \varepsilon 4 / \varepsilon 4 \varepsilon 4$ was associated with more than a $20 \%$ lower GCOG (Table 3).

Because GCOG represents a collection of somewhat dissociable cognitive domains, we next conducted a series of linear regression models to examine the relation of PBV and APOE to the level of function in five cognitive domains; episodic memory, visuospatial ability, semantic memory, perceptual speed, working memory. Interestingly, PBV was associated with better function in all five cognitive domains with its strongest effects on episodic memory (Table 3). Similarly, APOE $\varepsilon 3 \varepsilon 4 / \varepsilon 4 \varepsilon 4$ was associated with lower function in all five domains with its strongest effects on episodic memory (Table 3). By contrast, APOE $\varepsilon 2 \varepsilon 3$ was associated with better performance on tests of episodic memory and semantic memory (Table 3 ).

Of the variables determined to be independently associated with AD and cognition in this study, only HDL-R and PBV are potentially modifiable. We included HDL-R in our analyses based on the association of HDL-C [39] and TC [40] with AD, and also because of the association of APOE lipoprotein with HDL particles [41]. The relationship between these variables and the non-modifiable variables of age and $A P O E$ genotype on GCOG and the probability of dementia are graphically represented in Figure 1. The study population was normally distributed with respect to age, HDL-R, and PBV (Figure 1G-I) and the APOE $\varepsilon 2 \varepsilon 3 / / \varepsilon 3 \varepsilon 3 / / \varepsilon 3 \varepsilon 4 / \varepsilon 4 \varepsilon 4$ genotype distribution (13/64/23, Table 2) was similar to other published distributions [3]. The mean study GCOG was -0.03 and the overall study probability of dementia was $14.5 \%$. If cognition and dementia were considered as a continuum resulting from aging, relative to the $A P O E \varepsilon 3 \varepsilon 3$ genotype, the association of an $A P O E \varepsilon 3 \varepsilon 4 / \varepsilon 4 \varepsilon 4$ genotype with cognition or dementia was approximately equal to being older by 6.2 and 6.9 years, respectively, and a $A P O E \varepsilon 2 \varepsilon 3$ genotype was approximately equal to being younger by about 3.9 and 7.5 years of age, respectively (Figure 1C,F). Although a higher HDL-R is associated with higher cognition and a lower probability of dementia (Figure 1A,D), large differences in HDL-R are required to offset the associations attributable to $A P O E$ genotype. In contrast, relatively small differences in PBV are needed to offset the associations attributable to $A P O E$ genotype (Figure 1B,E). For example, the probability of dementia in $A P O E \varepsilon 3 \varepsilon 4 / \varepsilon 4 \varepsilon 4$ carriers with a PBV 0.3 units higher was equal to that of $A P O E \varepsilon 3 \varepsilon 3$ carriers, and with a PBV 0.6 units higher it was equal to that of $A P O E \varepsilon 2 \varepsilon 3$ carriers. Considering that 0.6 units represents less than $50 \%$ of the observed 1.3 unit PBV range, the biological system underlying PBV, if pharmacologically modifiable, has the potential to have a disease-modifying effect equal to or greater than the APOE genotype.

Modulation of the effects of age and $A P O E$ genotype on the probability of dementia by PBV is further illustrated in Figure 2. Probability of dementia approached zero irrespective of the genotype when the PBV index is numerically higher (Figure 2A). Similarly, while higher age increased dementia probability, the probability of dementia approached zero irrespective of the age at higher PBVs (Figure 2B). These results suggest that higher PBV is protective even when other risk factors are present. As expected, another well-known protective factor, the presence of $A P O E \varepsilon 2 \varepsilon 3$ allele, appeared to be also shielding against the effect of age on the probability of dementia (Figure $2 \mathrm{C}$ ). Consequently, APOE $\varepsilon 2 \varepsilon 3$ lowered the probability of dementia by an age equivalent to approximately 16 years compared to $A P O E \varepsilon 3 \varepsilon 4 / \varepsilon 4 \varepsilon 4$ carriers (Figure $2 \mathrm{C}$ ). In comparison, higher PBV (>1 SD), was protective against the effect of age on the probability of dementia by an age equivalent to approximately 14 years compared to lower PBV ( $<1 \mathrm{SD}$ ) (Figure 2D). 

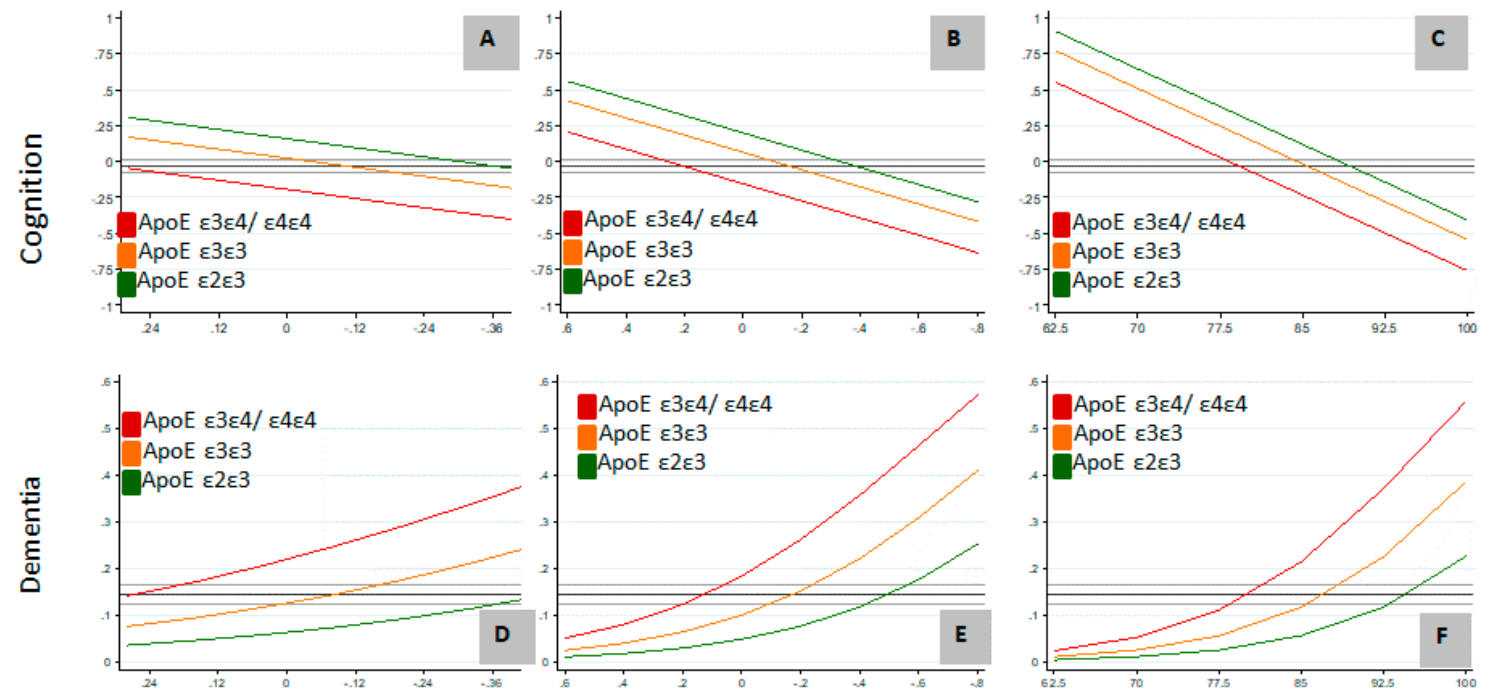

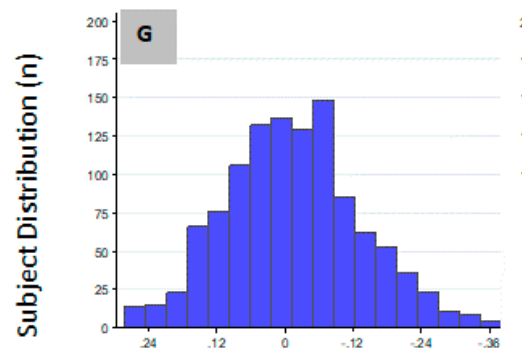

HDL-R

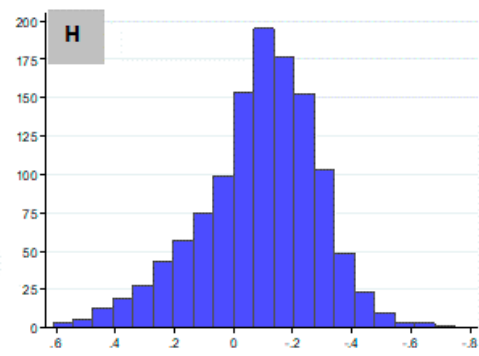

PBV

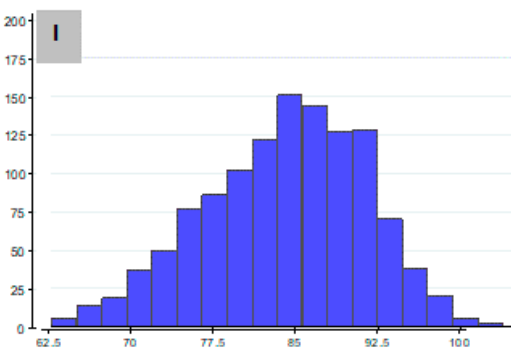

Age

Figure 1. The association of the distribution of High Density Lipoprotein (HDL) ratio (HDL-R), Plasmalogen Biosynthesis value (PBV) and age with probability of dementia and cognition in Apolipoprotein E (APOE) $\varepsilon 2 \varepsilon 3, \varepsilon 3 \varepsilon 3$ and $\varepsilon 3 \varepsilon 4 / \varepsilon 4 \varepsilon 4$ carriers. (A) Effect of HDL-R on cognition in different APOE genotypes; (B) Effect of PBV on cognition in different APOE genotypes; (C) Effect of age on cognition in different APOE genotypes; (D) Effect of HDL-R on probability of dementia in different APOE genotypes; (E) Effect of PBV on probability of dementia in different APOE genotypes; (F) Effect of age on probability of dementia in different APOE genotypes; (G) Distribution of HDL-R in the study cohort; (H) Distribution of PBV in the study cohort; (I) Distribution of age in the study cohort. Multiple regression analysis was carried out to determine the association of the distribution of HDL ratio, $\mathrm{PBV}$, and age with dementia and cognition in the study cohort. Cognition was measured as global cognition score (GCOG), which is the average of $z$-scores from a battery of cognitive measurements. Mean normalized, $\log 10$ transformed values of HDL-R, PBV, and mean-centered values of age are in the $x$-axes. $y$-axes represent GCOG in the graphs depicting the associations of cognition with HDL-R (A), PBV (B) and age (C); in the graphs depicting dementia (D-F), $y$-axes represent the probability of dementia as a fraction. In the distribution graphs $(\mathrm{G}-\mathrm{I}), y$-axes represent the number of individuals. HDL-R: HDL-C to total cholesterol ratio; PBV: Plasmalogen Biosynthesis value.

\subsection{Study Variable Associations on Serum Lipids}

The associations between the study variables and serum lipids are described in Table 4. Higher PBV was associated with lower TG, higher TC, higher LDL-C, higher HDL-C, and a higher HDL-R. A APOE $\varepsilon 2 \varepsilon 3$ genotype was associated with lower LDL-C and a higher HDL-R, and a APOE $\varepsilon 3 \varepsilon 4 / \varepsilon 4 \varepsilon 4$ genotype was associated with higher TC, higher LDL-C, and a lower HDL-R. No association between $A P O E$ genotype and PBV was observed. Of the study variables investigated, only PBV and $A P O E$ were associated with HDL-R levels (Table 4). 
Table 4. Multivariate association of clinical and biological variables with serum lipids.

\begin{tabular}{|c|c|c|c|c|c|}
\hline & $\begin{array}{l}\text { Total Cholesterol } \\
\quad(n=1085)\end{array}$ & $\begin{array}{l}\text { LDL-Cholesterol } \\
\qquad(n=1078)\end{array}$ & $\begin{array}{l}\text { HDL-Cholesterol } \\
\qquad(n=909)\end{array}$ & $\begin{array}{c}\text { HDL-R } \\
(n=909)\end{array}$ & $\begin{array}{l}\text { Trigylcerides } \\
(n=909)\end{array}$ \\
\hline Variable & Coef. ${ }^{1}(p)$ & Coef. ${ }^{1}(p)$ & Coef. $^{1}(p)$ & Coef. $^{1}(p)$ & Coef. $^{1}(p)$ \\
\hline PBV & $0.009\left(5.7 \times 10^{-4}\right)$ * & $0.014\left(2.6 \times 10^{-3}\right)$ * & $0.029\left(3.8 \times 10^{-15}\right)$ * & $0.020\left(5.0 \times 10^{-8}\right)$ * & $-0.043\left(3.8 \times 10^{-14}\right)$ * \\
\hline$A P O E \varepsilon 2 \varepsilon 3$ & $-0.009\left(2.8 \times 10^{-1}\right)$ & $-0.036\left(7.6 \times 10^{-3}\right) *$ & $0.018\left(9.7 \times 10^{-2}\right)$ & $0.027\left(1.3 \times 10^{-2}\right) *$ & $0.014\left(4.2 \times 10^{-1}\right)$ \\
\hline$A P O E \varepsilon 3 \varepsilon 4, \varepsilon 4 \varepsilon 4$ & $0.021\left(9.7 \times 10^{-4}\right)^{*}$ & $0.042\left(1.2 \times 10^{-4}\right)^{*}$ & $-0.002\left(8.1 \times 10^{-1}\right)$ & $-0.023\left(6.5 \times 10^{-3}\right) *$ & $0.002\left(8.8 \times 10^{-1}\right)$ \\
\hline Age & $-0.009\left(1.1 \times 10^{-3}\right) *$ & $-0.014\left(2.0 \times 10^{-3}\right) *$ & $-0.002\left(5.6 \times 10^{-1}\right)$ & $0.007\left(6.1 \times 10^{-2}\right)$ & $-0.006\left(2.6 \times 10^{-1}\right)$ \\
\hline Female & Reference & Reference & Reference & Reference & Reference \\
\hline Male & $-0.001\left(8.5 \times 10^{-1}\right)$ & $-0.001\left(9.4 \times 10^{-1}\right)$ & $-0.002\left(8.1 \times 10^{-1}\right)$ & $-0.001\left(8.7 \times 10^{-1}\right)$ & $0.007\left(6.3 \times 10^{-1}\right)$ \\
\hline
\end{tabular}

Multiple regression analysis was carried out to determine the associations of plasmalogen biosynthesis value (PBV), $A P O E$ genotype, age, education, and gender with serum lipid parameters. ${ }^{1}$ Coefficients for continuous variables expressed per standard deviation (SD); Age and Education were mean centered; Lipids were mean normalized then $\log _{10}$ transformed. For clarity, significant $p$ values $(p<0.05)$ are indicated by an asterisk *
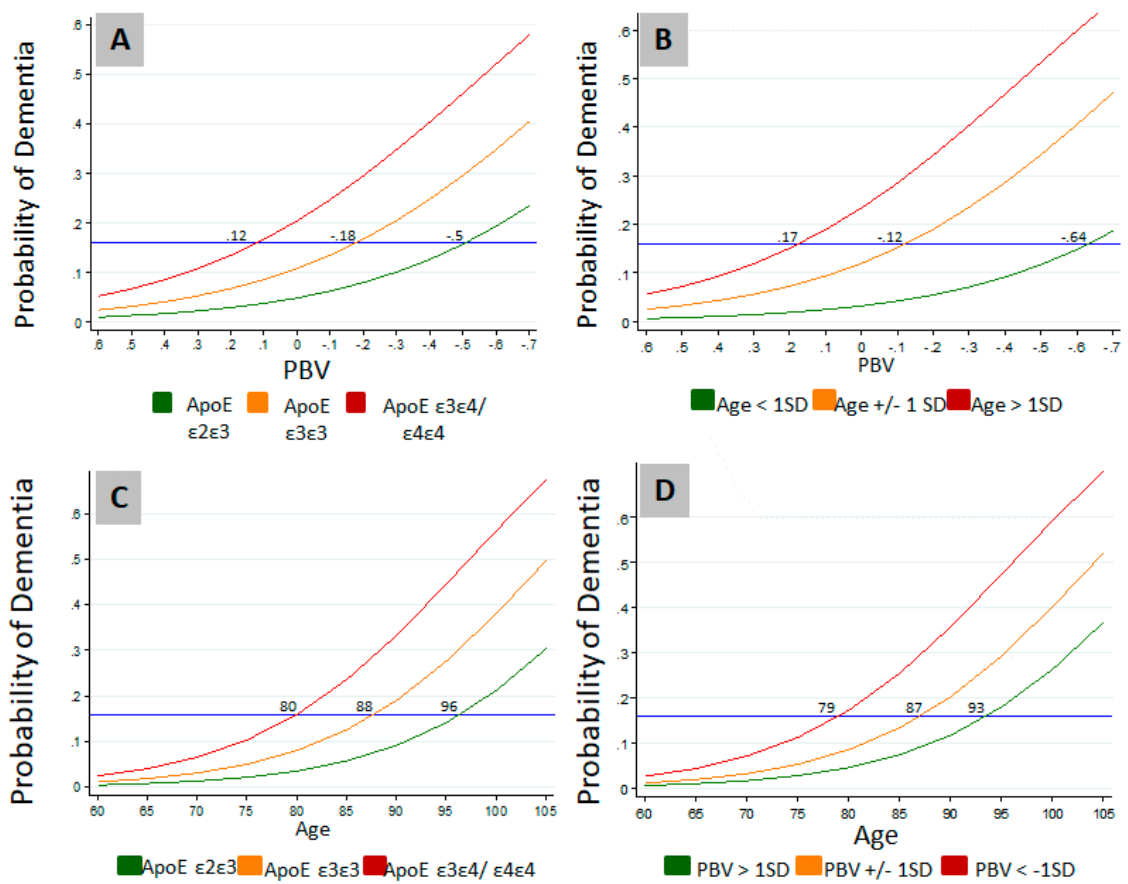

Figure 2. Effects of age and APOE genotype on the probability of dementia is modulated by PBV. (A) Modulation of the effect of genotype on the probability of dementia by PBV; (B) Effects of age distribution on the probability of dementia at different PBV levels; (C) Effects of genotype on the probability of dementia at different ages; (D) Effects of PBV distribution on the probability of dementia at different ages. Logistic regression was carried out to determine the effects of PBV, age, and APOE genotype on the probability of dementia in the study cohort. PBV: Plasmalogen Biosynthesis Value; SD: Standard Deviation.

\section{Discussion}

Age and $A P O E$ genotype are the primary variables associated with the probability of AD and the observations of this study support these well accepted facts. The importance of understanding the biochemical pathway(s) upon or within which advancing age or the ApoE isoforms interact to effect changes in cognition and cause AD cannot be overstated. As an inborn genetic risk factor, $A P O E$ genotype is, de facto, within the causative cascade of AD. The ApoE isoform association with disease prevalence $(\varepsilon 4>\varepsilon 3>\varepsilon 2)$ [3] is analogous to a medicinal chemistry structure-activity relationship and suggestive of a quantitative and continuous underlying mechanism. The isoform-specific prevalence rates further suggest that if this mechanism were to be found and successfully targeted, that AD 
prevalence could be reduced to at least that of the $\varepsilon 2 \varepsilon 3$ genotype. This would result in an overall reduction in $\mathrm{AD}$ cases by $75 \%$ or more [42].

The two prevailing ApoE hypotheses involve interactions with amyloid and/or cholesterol regulation and homeostasis and a third, less studied mechanism involves altered membrane composition. All three are interrelated biochemically. ApoE has been shown to exert an isoform-specific causal effect on amyloid accumulation in both imaging $[43,44]$ and post-mortem $[45,46]$ studies, and membrane PlsEtn enrichment has been shown to dose-dependently decrease $A \beta_{1-42}$ via increased $\alpha$-secretase [47]. However, the hypothesis that amyloid accumulation causes a decline in cognition remains unproven. To the contrary, anti-amyloid therapies have consistently failed to affect cognition in humans [48].

Age is the primary variable associated with the probability of $\mathrm{AD}$ and the observations of this study support this well-accepted fact. The distributions of age, cognitive status, and diagnosis of $\mathrm{AD}$ within this study allowed us to robustly investigate the associations between non-modifiable variables, such as age and $A P O E$ genotype, and putatively modifiable metabolic covariates with $\mathrm{AD}$ and cognition. After correcting for APOE genotype and demographic variables, biomarkers of PBV and the ratio of HDL-R were independently associated with cognition and dementia with PBV having a magnitude of association similar to that of $A P O E$ genotype.

Although no direct interactions between PBV and APOE were observed, both the protective APOE $\varepsilon 2 \varepsilon 3$ genotype and high (protective) levels of PBV were associated with higher HDL-R (Table 4 ) and higher HDL-R was independently associated with higher cognition and a lower odds of dementia (Table 3). Since HDL-R is a measure of HDL-MCE, these observations suggest that both APOE and PlsEtn may be interacting with HDL-MCE via independent mechanisms.

The role of cholesterol dysregulation and APOE in AD has been recently reviewed [18,19]. Higher levels of free cholesterol in human post-mortem cortical membranes has been associated with lower cognition [49]; lower total cholesterol in the Cerebrospinal fluid (CSF) of AD patients is suggestive of lower cholesterol efflux in AD [50,51]; and increasing membrane cholesterol reportedly induces the $\beta$-secretase pathway and the accumulation of amyloid independent of APOE genotype [52]. $A P O E$ has been shown to exert an isoform-specific causal effect on amyloid accumulation in both imaging $[43,44]$ and post-mortem $[45,46]$ studies, and membrane PlsEtn enrichment has been shown to dose-dependently decrease $A \beta_{1-42}$ via increased $\alpha$-secretase [47]. However, the hypothesis that amyloid accumulation causes a decline in cognition remains to be proven clinically [48].

The clinical implications of this study are obvious. This is the first reported evidence of a metabolic phenotype with the same clinical characteristics as the APOE $\varepsilon 2 \varepsilon 3$ genotype. The probability of dementia in participants with either a high PBV or an APOE $\varepsilon 2 \varepsilon 3$ genotype was indistinguishable. Accordingly, effective pharmacological targeting of the plasmalogen biosynthesis pathway has the potential of having a disease-modifying effect in $\mathrm{AD}$ equal to or greater than that of the APOE $\varepsilon 2 \varepsilon 3$ genotype.

\section{Conclusions}

The key conclusions of this study are that lower serum PBV levels are associated with higher odds of MCI/AD and with lower levels of cognition. Higher PBV levels ( $>1 \mathrm{SD}$ ) appeared to modulate the negative effects of age and APOE $\varepsilon 4$ allele on the probability of dementia. This study, thus, identifies lower levels of PBV as a potentially modifiable risk factor for dementia. This finding would, potentially, pave the way for interventions that can lower the risk of dementia in high-risk individuals.

Author Contributions: Conceptualization, D.B.G.; Methodology, D.B.G.; Experimental supervision, D.B.G; Data analysis and interpretation, D.B.G; Writing-original draft preparation, D.B.G.; Writing-review and editing, D.B.G and V.S. All authors read and approved the final version of the manuscript.

Funding: This research received no external funding. 
Acknowledgments: We wish to thank David A. Bennett and Sue E. Leurgans for critical review of the manuscript and for their valuable suggestions. We acknowledge and thank Rush University Alzheimer's Disease Center for providing the serum samples and access to ROS/MAP data.

Conflicts of Interest: The corresponding author is the CEO and President of Prodrome Sciences Inc., and was the former CEO and President of Phenomenome Discoveries Inc. Second author is an employee of Prodrome Sciences Inc. and was an employee of Phenomenome Discoveries Inc.

\section{References}

1. Pitas, R.E.; Boyles, J.K.; Lee, S.H.; Hui, D.; Weisgraber, K.H. Lipoproteins and their receptors in the central nervous system. Characterization of the lipoproteins in cerebrospinal fluid and identification of apolipoprotein B,E(LDL) receptors in the brain. J. Biol. Chem. 1987, 262, 14352-14360. [PubMed]

2. Corder, E.H.; Saunders, A.M.; Strittmatter, W.J.; Schmechel, D.E.; Gaskell, P.C.; Small, G.W.; Roses, A.D.; Haines, J.L.; Pericak-Vance, M.A. Gene dose of apolipoprotein E type 4 allele and the risk of Alzheimer's disease in late onset families. Science 1993, 261, 921-923. [CrossRef] [PubMed]

3. Ashford, J.W. APOE genotype effects on Alzheimer's disease onset and epidemiology. J. Mol. Neurosci. 2004, 23, 157-165. [CrossRef]

4. Hauser, P.S.; Narayanaswami, V.; Ryan, R.O. Apolipoprotein E: from lipid transport to neurobiology. Prog. Lipid Res. 2011, 50, 62-74. [CrossRef]

5. Kim, J.; Basak, J.M.; Holtzman, D.M. The role of apolipoprotein E in Alzheimer's disease. Neuron 2009, 63, 287-303. [CrossRef] [PubMed]

6. Goodenowe, D.B.; Cook, L.L.; Liu, J.; Lu, Y.; Jayasinghe, D.A.; Ahiahonu, P.W.; Heath, D.; Yamazaki, Y.; Flax, J.; Krenitsky, K.F.; et al. Peripheral ethanolamine plasmalogen deficiency: a logical causative factor in Alzheimer's disease and dementia. J. Lipid Res. 2007, 48, 2485-2498. [CrossRef] [PubMed]

7. Wood, P.L.; Mankidy, R.; Ritchie, S.; Heath, D.; Wood, J.A.; Flax, J.; Goodenowe, D.B. Circulating plasmalogen levels and Alzheimer Disease Assessment Scale-Cognitive scores in Alzheimer patients. J. Psychiatry Neurosci. 2010, 35, 59-62. [CrossRef]

8. Chang, C.-Y.; Ke, D.-S.; Chen, J.-Y. Essential fatty acids and human brain. Acta Neurol. Taiwanica 2009, 18, 231-241.

9. Farooqui, A.A.; Farooqui, T.; Horrocks, L.A. Metabolism and Functions of Bioactive Ether Lipids in the Brain; Springer Nature: New York, NY, USA, 2008.

10. Rouser, G.; Yamamoto, A. Curvilinear regression course of human brain lipid composition changes with age. Lipids 1968, 3, 284-287. [CrossRef]

11. Ginsberg, L.; Rafique, S.; Xuereb, J.H.; Rapoport, S.I.; Gershfeld, N.L. Disease and anatomic specificity of ethanolamine plasmalogen deficiency in Alzheimer's disease brain. Brain Res. 1995, 698, 223-226. [CrossRef]

12. Han, X.; Holtzman, D.M.; McKeel, D.W., Jr. Plasmalogen deficiency in early Alzheimer's disease subjects and in animal models: molecular characterization using electrospray ionization mass spectrometry. J. Neurochem. 2001, 77, 1168-1180. [CrossRef]

13. Guan, Z.; Wang, Y.; Cairns, N.J.; Lantos, P.L.; Dallner, G.; Sindelar, P.J. Decrease and Structural Modifications of Phosphatidylethanolamine Plasmalogen in the Brain with Alzheimer Disease. J. Neuropathol. Exp. Neurol. 1999, 58, 740-747. [CrossRef] [PubMed]

14. Farooqui, A.A.; Rapoport, S.I.; Horrocks, L.A. “Membrane Phospholipid Alterations in Alzheimer's Disease: Deficiency of Ethanolamine Plasmalogens. Neurochem. Res. 1997, 22, 523-527. [CrossRef]

15. Braverman, N.E.; Moser, A.B. Functions of plasmalogen lipids in health and disease. Biochim. Biophys. Acta 2012, 1822, 1442-1452. [CrossRef]

16. Bennett, D.A.; Schneider, J.A.; Aggarwal, N.T.; Arvanitakis, Z.; Shah, R.C.; Kelly, J.F.; Fox, J.H.; Cochran, E.J.; Arends, D.; Treinkman, A.D.; et al. Decision rules guiding the clinical diagnosis of Alzheimer's disease in two community-based cohort studies compared to standard practice in a clinic-based cohort study. Neuroepidemiology 2006, 27, 169-176. [CrossRef]

17. Knopman, D.S.; DeKosky, S.T.; Cummings, J.L.; Chui, H.; Corey-Bloom, J.; Relkin, N.; Small, G.W.; Miller, B.; Stevens, J.C. Practice Parameter: Diagnosis of Dementia (an Evidence-Based Review). Report of the Quality Standards Subcommittee of the American Academy of Neurology. Neurology 2001, 56, 1143-1153. [CrossRef] [PubMed] 
18. Poirier, J.; Miron, J.; Picard, C.; Gormley, P.; Theroux, L.; Breitner, J.; Dea, D. Apolipoprotein E and lipid homeostasis in the etiology and treatment of sporadic Alzheimer's disease. Neurobiol. Aging 2014, 35, 3-10. [CrossRef]

19. Leoni, V.; Solomon, A.; Kivipelto, M. Links between ApoE, brain cholesterol metabolism, tau and amyloid beta-peptide in patients with cognitive impairment. Biochem. Soc. Trans. 2010, 38, 1021-1025. [CrossRef] [PubMed]

20. Michikawa, M.; Fan, Q.W.; Isobe, I.; Yanagisawa, K. Apolipoprotein E exhibits isoform-specific promotion of lipid efflux from astrocytes and neurons in culture. J. Neurochem. 2000, 74, 1008-1016. [CrossRef] [PubMed]

21. Munn, N.J.; Arnio, E.; Liu, D.; Zoeller, R.A.; Liscum, L. Deficiency in ethanolamine plasmalogen leads to altered cholesterol transport. J. Lipid Res. 2003, 44, 182-192. [CrossRef] [PubMed]

22. Mankidy, R.; Ahiahonu, P.W.; Ma, H.; Jayasinghe, D.; Ritchie, S.A.; Khan, M.A.; Su-Myat, K.K.; Wood, P.L.; Goodenowe, D.B. Membrane plasmalogen composition and cellular cholesterol regulation: A structure activity study. Lipids Health Dis. 2010, 9, 9-62. [CrossRef]

23. Mandel, H.; Sharf, R.; Berant, M.; Wanders, R.J.; Vreken, P.; Aviram, M. Plasmalogen phospholipids are involved in HDL-mediated cholesterol efflux: Insights from investigations with plasmalogen-deficient cells. Biochem. Biophys. Res. Comm. 1998, 250, 369-373. [CrossRef]

24. Oikawa, N.; Hatsuta, H.; Murayama, S.; Suzuki, A.; Yanagisawa, K. Influence of APOE genotype and the presence of Alzheimer's pathology on synaptic membrane lipids of human brains. J. Neurosci. Res. 2014, 92, 641-650. [CrossRef]

25. Igbavboa, U.; Eckert, G.P.; Malo, T.M.; Studniski, A.E.; Johnson, L.N.; Yamamoto, N.; Kobayashi, M.; Fujita, S.C.; Appel, T.R.; Muller, W.E.; et al. Murine synaptosomal lipid raft protein and lipid composition are altered by expression of human Apoe 3 and 4 and by increasing age. J. Neurol. Sci. 2005, 229-230, 225-232. [CrossRef] [PubMed]

26. Johnson, L.A.; Olsen, R.H.; Merkens, L.S.; Debarber, A.; Steiner, R.D.; Sullivan, P.M.; Maeda, N.; Raber, J. Apolipoprotein E-low density lipoprotein receptor interaction affects spatial memory retention and brain ApoE levels in an isoform-dependent manner. Neurobiol. Dis. 2014, 64, 150-162. [CrossRef] [PubMed]

27. Bennett, D.A.; Schneider, J.A.; Arvanitakis, Z.; Wilson, R.S. Overview and findings from the religious orders study. Curr. Alzheimer. Res. 2012, 9, 628-645. [CrossRef] [PubMed]

28. Bennett, D.A.; Schneider, J.A.; Buchman, A.S.; Barnes, L.L.; Boyle, P.A.; Wilson, R.S. Overview and findings from the rush Memory and Aging Project. Curr. Alzheimer. Res. 2012, 9, 646-663. [CrossRef]

29. Bennett, D.A.; Schneider, J.A.; Buchman, A.S.; Mendes de Leon, C.; Bienias, J.L.; Wilson, R.S. The Rush Memory and Aging Project: Study design and baseline characteristics of the study cohort. Neuroepidemiology 2005, 25, 163-175. [CrossRef]

30. Wilson, R.S.; Leurgans, S.E.; Boyle, P.A.; Bennett, D.A. Cognitive decline in prodromal Alzheimer disease and mild cognitive impairment. Arch. Neurol. 2011, 68, 351-356. [CrossRef]

31. Bennett, D.A.; Wilson, R.S.; Schneider, J.A.; Evans, D.A.; Beckett, L.A.; Aggarwal, N.T.; Barnes, L.L.; Fox, J.H.; Bach, J. Natural history of mild cognitive impairment in older persons. Neurology 2002, 59, 198-205. [CrossRef]

32. Hagve, T.A.; Christophersen, B.O. Evidence for peroxisomal retroconversion of adrenic acid (22:4(n-6)) and docosahexaenoic acids (22:6(n-3)) in isolated liver cells. Biochim. Biophys. Acta 1986, 875, 165-173. [CrossRef]

33. Gronn, M.; Christensen, E.; Hagve, T.A.; Christophersen, B.O. Peroxisomal retroconversion of docosahexaenoic acid (22:6(n-3)) to eicosapentaenoic acid (20:5(n-3)) studied in isolated rat liver cells. Biochim. Biophys. Acta 1991, 1081, 85-91. [CrossRef]

34. Moore, S.A.; Hurt, E.; Yoder, E.; Sprecher, H.; Spector, A.A. Docosahexaenoic acid synthesis in human skin fibroblasts involves peroxisomal retroconversion of tetracosahexaenoic acid. J. Lipid Res. 1995, 36, 2433-2443. [PubMed]

35. Olazaran, J.; Gil-de-Gomez, L.; Rodriguez-Martin, A.; Valenti-Soler, M.; Frades-Payo, B.; Marin-Munoz, J.; Antunez, C.; Frank-Garcia, A.; Acedo-Jimenez, C.; Morlan-Gracia, L.; et al. A blood-based, 7-metabolite signature for the early diagnosis of Alzheimer's disease. J. Alzheimers Dis. 2015, 45, 1157-1173. [CrossRef] [PubMed]

36. Abdullah, L.; Evans, J.E.; Emmerich, T.; Crynen, G.; Shackleton, B.; Keegan, A.P.; Luis, C.; Tai, L.; LaDu, M.J.; Mullan, M.; et al. APOE epsilon4 specific imbalance of arachidonic acid and docosahexaenoic acid in serum phospholipids identifies individuals with preclinical Mild Cognitive Impairment/Alzheimer's Disease. Aging 2017, 9, 964-985. [CrossRef] 
37. Nishimukai, M.; Maeba, R.; Yamazaki, Y.; Nezu, T.; Sakurai, T.; Takahashi, Y.; Hui, S.P.; Chiba, H.; Okazaki, T.; Hara, H. Serum choline plasmalogens, particularly those with oleic acid in sn-2, are associated with proatherogenic state. J. Lipid Res. 2014, 55, 956-965. [CrossRef]

38. Dorninger, F.; Brodde, A.; Braverman, N.E.; Moser, A.B.; Just, W.W.; Forss-Petter, S.; Brugger, B.; Berger, J. Homeostasis of phospholipids-The level of phosphatidylethanolamine tightly adapts to changes in ethanolamine plasmalogens. Biochim. Biophys. Acta 2015, 1851, 117-128. [CrossRef]

39. Koch, M.; Jensen, M.K. HDL-cholesterol and apolipoproteins in relation to dementia. Curr. Opin. Lipidol. 2016, 27, 76-87. [CrossRef] [PubMed]

40. Anstey, K.J.; Ashby-Mitchell, K.; Peters, R. Updating the Evidence on the Association between Serum Cholesterol and Risk of Late-Life Dementia: Review and Meta-Analysis. J. Alzheimers Dis. 2017, 56, 215-228. [CrossRef]

41. Getz, G.S.; Reardon, C.A. Apoprotein E as a lipid transport and signaling protein in the blood, liver, and artery wall. J. Lipid Res. 2009, 50 Suppl, S156-S161. [CrossRef]

42. Raber, J.; Huang, Y.; Ashford, J.W. ApoE genotype accounts for the vast majority of AD risk and AD pathology. Neurobiol. Aging 2004, 25, 641-650. [CrossRef]

43. Kim, H.J.; Ye, B.S.; Yoon, C.W.; Cho, H.; Noh, Y.; Kim, G.H.; Choi, Y.S.; Kim, J.H.; Jeon, S.; Lee, J.M.; et al. Effects of APOE epsilon4 on brain amyloid, lacunar infarcts, and white matter lesions: A study among patients with subcortical vascular cognitive impairment. Neurobiol. Aging 2013, 34, 2482-2487. [CrossRef]

44. Morris, J.C.; Roe, C.M.; Xiong, C.; Fagan, A.M.; Goate, A.M.; Holtzman, D.M.; Mintun, M.A. APOE predicts amyloid-beta but not tau Alzheimer pathology in cognitively normal aging. Ann. Neurol. 2010, 67, 122-131. [CrossRef] [PubMed]

45. Caselli, R.J.; Walker, D.; Sue, L.; Sabbagh, M.; Beach, T. Amyloid load in nondemented brains correlates with APOE e4. Neurosci. Lett. 2010, 473, 168-171. [CrossRef] [PubMed]

46. Bennett, D.A.; Wilson, R.S.; Schneider, J.A.; Evans, D.A.; Aggarwal, N.T.; Arnold, S.E.; Cochran, E.J.; Berry-Kravis, E.; Bienias, J.L. Apolipoprotein E epsilon4 allele, AD pathology, and the clinical expression of Alzheimer's disease. Neurology 2003, 60, 246-252. [CrossRef]

47. Wood, P.L.; Khan, A.M.; Mankidy, R.; Smith, T.; Goodenowe, D. Plasmalogen Deficit: A New and Testable Hypothesis for the Etiology of Alzheimer's Disease, Alzheimer's Disease Pathogenesis-Core Concepts, Shifting Paradigms and Therapeutic Targets, Suzanne De La Monte, IntechOpen. Available online: https:/www.intechopen.com/books/alzheimer-s-disease-pathogenesis-core-concepts-shiftingparadigms-and-therapeutic-targets/plasmalogen-deficit-a-new-and-testable-hypothesis-for-the-etiologyof-alzheimer-s-disease (accessed on 22 April 2019).

48. Castello, M.A.; Jeppson, J.D.; Soriano, S. Moving beyond anti-amyloid therapy for the prevention and treatment of Alzheimer's disease. BMC Neurol. 2014, 14, 169. [CrossRef]

49. Cutler, R.G.; Kelly, J.; Storie, K.; Pedersen, W.A.; Tammara, A.; Hatanpaa, K.; Troncoso, J.C.; Mattson, M.P. Involvement of oxidative stress-induced abnormalities in ceramide and cholesterol metabolism in brain aging and Alzheimer's disease. Proc. Natl. Acad. Sci. USA 2004, 101, 2070-2075. [CrossRef] [PubMed]

50. Yamauchi, K.; Tozuka, M.; Nakabayashi, T.; Sugano, M.; Hidaka, H.; Kondo, Y.; Katsuyama, T. Apolipoprotein E in cerebrospinal fluid: relation to phenotype and plasma apolipoprotein E concentrations. Clin. Chem. 1999, 45, 497-504.

51. Mulder, M.; Ravid, R.; Swaab, D.F.; de Kloet, E.R.; Haasdijk, E.D.; Julk, J.; van der Boom, J.J.; Havekes, L.M. Reduced levels of cholesterol, phospholipids, and fatty acids in cerebrospinal fluid of Alzheimer disease patients are not related to apolipoprotein E4. Alzheimer Dis. Assoc. Disord. 1998, 12, 198-203. [CrossRef]

52. Ghribi, O.; Larsen, B.; Schrag, M.; Herman, M.M. High cholesterol content in neurons increases BACE, beta-amyloid, and phosphorylated tau levels in rabbit hippocampus. Exp. Neurol. 2006, 200, 460-467. [CrossRef]

(C) 2019 by the authors. Licensee MDPI, Basel, Switzerland. This article is an open access article distributed under the terms and conditions of the Creative Commons Attribution (CC BY) license (http://creativecommons.org/licenses/by/4.0/). 